\title{
Light penetration in the human prostate: a whole prostate clinical study at $763 \mathrm{~nm}$
}

\author{
Caroline M. Moore, ${ }^{\text {a,b }}$ C. Alexander Mosse, ${ }^{a}$ Clare Allen, ${ }^{c}$ Heather Payne, ${ }^{d}$ Mark Emberton, ${ }^{b}$ and Stephen G. Bown ${ }^{a}$ \\ ${ }^{a}$ National Medical Laser Centre, University College London, London, W1W 7EJ United Kingdom \\ ${ }^{\mathrm{b}}$ Department of Urology, University College London Hospitals Trust, London, NW1 2PG, United Kingdom \\ ${ }^{c}$ Department of Imaging, University College London Hospitals Trust, London, NW1 2PG, United Kingdom \\ ${ }^{\mathrm{d} C l i n i c a l}$ Oncology Department, University College London Hospitals Trust, London, NW1 2PG, United Kingdom
}

\begin{abstract}
Photodynamic therapy (PDT) is being investigated as a treatment for localized prostate cancer. Photodynamic therapy uses a photosensitizing drug which is activated by a specific wavelength of light, in the presence of oxygen. The activated drug reacts with tissue oxygen to produce reactive oxygen species which are responsible for localized tissue necrosis. One of the determinants of the PDT effect is the penetration of light in the prostate. This study assesses the penetration depth of $763 \mathrm{~nm}$ light throughout the prostate. Eight men undergoing multiple hollow needle insertion for high dose rate brachytherapy were recruited. $763 \mathrm{~nm}$ light, produced by a diode laser, was delivered to the prostate using cylindrically diffusing optical fibers within the plastic needles. Light was detected at different distances from the source, using an isotropic detector within nearby needles. Penetration depth was calculated using the Boltzmann approximation to the diffusion equation. Delivery detector fiber separation was measured on computed tomography. The mean penetration depth was $0.57 \mathrm{~cm}$, but there was within patient variation of a mean factor of 4.3. Further work is ongoing to assess the effect of such variability in light penetration, on the PDT effect. (C) 2011 Society of Photo-Optical Instrumentation Engineers (SPIE). [DOI: 10.1117/1.3528638]
\end{abstract}

Keywords: photodynamic therapy; light penetration; prostate; prostate cancer.

Paper 10195SSR received Apr. 13, 2010; revised manuscript received Nov. 24, 2010; accepted for publication Nov. 29, 2010; published online Jan. 27, 2011.

\section{Introduction}

Prostate cancer is the most common cancer diagnosed in men in the UK, and the second leading cause of cancer death in men in the USA. ${ }^{1}$ It accounts for 1 in 5 of all male cancers. With prostate specific antigen (PSA) testing becoming widespread, it is often diagnosed at an early stage when it is confined to the prostate. Current treatments include surgery and radiotherapy. Each of these can have significant side effects, particularly in terms of continence and potency. Minimally invasive treatments are under investigation for the treatment of organ confined prostate cancer. These include cryotherapy, high intensity focused ultrasound, and photodynamic therapy.

We are undertaking clinical studies of photodynamic therapy (PDT) for prostate cancer which will be reported elsewhere. PDT uses a photosensitising drug, given intravenously, and then activated by light of a specific wavelength, in the presence of oxygen. For these clinical PDT studies the light is delivered to the prostate using cylindrically diffusing fibers, placed within hollow, translucent, plastic needles.

One of the determinants of the photodynamic effect is the fluence - that is, the light available to activate the photosensitizer in any given volume of tissue. This is determined by the energy delivered to the tissue and the translucency of the tissue. The translucency for any particular wavelength can be thought of in terms of penetration depth, which is defined as the distance from the source at which $67 \%$ of the light fluence is lost.

Address all correspondence to: Caroline M. Moore, Clinical Lecturer in Urology, University College London, $3^{\text {rd }}$ Floor, Charles Bell House, 6773 Riding House Street, London W1W7EJ, United Kingdom. E-mail: carolinemoore@doctors.org.uk.
The penetration depth is known to vary with wavelength of light, and between tissues. Previous studies have assessed differences in light penetration between benign and malignant tissue, between the right and left lobes of the prostate, and between 633 and $665 \mathrm{~nm}$ light. ${ }^{2,3}$ It was concluded that there was no significant difference between benign and malignant tissue; that $665 \mathrm{~nm}$ light had a greater penetration depth than $633 \mathrm{~nm}$; and that there could be up to $60 \%$ difference in penetration depth between the right and left lobes in the same patient. It was proposed that penetration depth be measured in each of the right and left lobes of the prostate prior to light delivery for PDT in order to deliver a light dose based on tissue translucency in that lobe.

A further study by Lee used a transperineal approach with seven patients and found a twofold variation in penetration depth $\left(1 / \mu_{\text {eff }}\right.$, where $\mu_{\text {eff }}$ is the effective attenuation coefficient) at $630 \mathrm{~nm} .{ }^{4}$ Interestingly, they found that their measurements implied a 10 -fold variation in the intensity of the light emitting fiber although it had been calibrated before being inserted into the tissues. They suggested that this might be due to blood pooling around some of the fibers and absorbing light before it could enter the tissues whose optical properties they were investigating.

Until the mid 1990's clinical measurements of light penetration in the prostate had been limited to two or three positions per prostate, in men undergoing prostate biopsy or transurethral resection of the prostate. Since clinical studies started evaluating prostate PDT in the mid 1990's, men undergoing PDT for prostate cancer have had light measurements at multiple

1083-3668/2011/16(1)/015003/12/\$25.00 @ 2011 SPIE 
positions in the prostate ${ }^{5-7}$ The first studies by $\mathrm{Zhu}^{5}$ used an optimisation method to extract optical properties based on the measurement of fluence rate at several distances from a point source. Use of a motorized probe allowed the taking of a high number of readings in a short time. Because of the greater number of measurements available a modified differential evolution algorithm was used to fit the measured data using the diffusion approximation, and a map of optical properties was made by fitting each source detector pair individually. Zhu terms this a point-by-point method. ${ }^{8}$ Later work from Zhu and colleagues has used interstitial diffuse optical tomography to reconstruct optical properties from optical measurements made in men undergoing PDT using motexafin lutetium, following failure of radiotherapy. ${ }^{6}$ As described the process would take too long to be practical for use in treatment planning.

The photosensitizer in the prostate is another factor which would affect the measured optical properties. Other groups have taken measurements prior to or during photodynamic therapy for radio recurrent prostate cancer, where there is photosensitizer in the prostate and in some cases photosensitizer distribution itself showed heterogeneity. ${ }^{9-13}$ The photosensitizer planned for use in our clinical work is a vascular acting photosensitizer (Tookad, WST-09, Steba Biotech, France) which is activated by $763 \mathrm{~nm}$ light a short time after infusion (10- $20 \mathrm{~min})$. It would not, therefore, be possible to measure optical properties with the photosensitizer on board and then deliver a therapeutic light dose based on these measurements.

We report our assessment of light penetration in men who have not received any photosensitizer. The study population comprised men having high dose rate brachytherapy for prostate cancer, who have up to 24 needles in the prostate for a $30 \mathrm{~h}$ period, allowing access for multiple light measurements throughout the prostate. The aim of the work reported here was to assess whether the optical equipment used for the clinical work could be used to assess the optical properties of the prostate, and hence be used in treatment planning.

\subsection{Patients and Methods}

The study was submitted to and approved by the Joint University College London, University College London Hospitals Foundation Trust (UCLH) Committee on the Ethics of Human Research. Patients having high dose rate brachytherapy under the care of Dr. Heather Payne at University College London Hospitals Trust were approached to take part in the study.

\section{High Dose Rate Brachytherapy}

High dose rate (HDR) brachytherapy for prostate cancer involves the insertion of between 12 and 24 hollow, translucent plastic needles with closed ends (5Fr Nucletron prostate brachytherapy needles, Nucletron, Cheshire, UK) into the prostate under general anaesthetic. The radiotherapy dose is then delivered in two or three fractions directly into the prostate. At UCLH it is used for men with high risk prostate cancer (either PSA $>10$, Gleason sum 7 or locally advanced disease on imaging). HDR brachytherapy is given as a boost prior to a course of external beam therapy, and in conjunction with 2 years of hormone therapy, which is started 3-6 months prior to the brachytherapy.

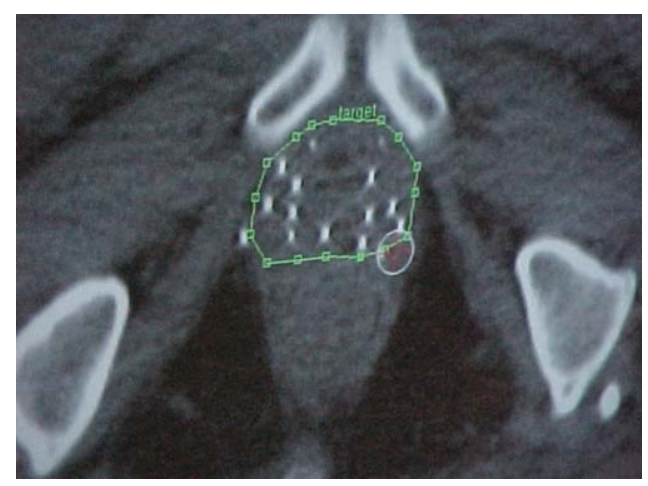

Fig. 1 CT image of brachytherapy needles in position.

Needles are inserted into the prostate with the aid of transrectal ultrasound and a perineal template. The template holes are labeled according to coordinates with the 1 row being the row closest to the rectum, and higher rows labelled 1.5, 2, 2,5, etc. The columns are labeled with capital and lower case letters, from the patients right to left, i.e., A,a,B,b, etc., with the urethra on the $\mathrm{d}$ column. Once all the needle positions are determined to be satisfactory the template is locked, (to prevent the needles from moving with respect to the template), and the template sewn to the perineum.

Following recovery from the general anaesthetic the patient has a computed tomography (CT) scan of the prostate, and these images are transferred to the radiotherapy planning department, for use in treatment planning (Fig. 1).

The study was carried out following needle insertion, prior to delivery of the first dose of radiotherapy.

\section{Equipment}

Light was delivered to the prostate using a 2-cm cylindrical diffuser (model CD 403-20, Ceramoptec, Bonn, Germany) and a 763-nm diode laser (Ceralas PDT 763 nm 4W: Ceramoptec). A cylindrical diffuser, rather than a point source, was used as this is the usual method of light delivery for clinical interstitial PDT. Light was detected with an isotropic light detector ("Squere", Resonance Optics, South Yarmouth, MA), connected to a Newport optical power meter (Newport Corporation, Irvine, CA) (Fig. 2).

The Squere uses a 12-mm-long by 1-mm-wide bar-shaped detector end, rather than the more commonly seen golf ball tip. This was necessary in this study because the golf ball tip detector available at the time did not fit into the brachytherapy needles. In common with other small isotropic detectors, the detector in the Squere is shadowed by the optical fiber it is attached to but otherwise has uniform sensitivity $( \pm 10 \%$ over $260^{\circ}$, manufacturer's data). The Ceramoptec data sheet shows that the cylindrically diffusing fiber has a uniform irradiance $( \pm 3 \%)$ over the central $15 \mathrm{~mm}$ of its length with the intensity falling off either side of this (Fig. 3).

Although the brachytherapy needles are not designed for optical use, they are translucent and measurements made using the integrating sphere incorporated into the laser showed that they absorb less than $5 \%$ of the light emitted from the 


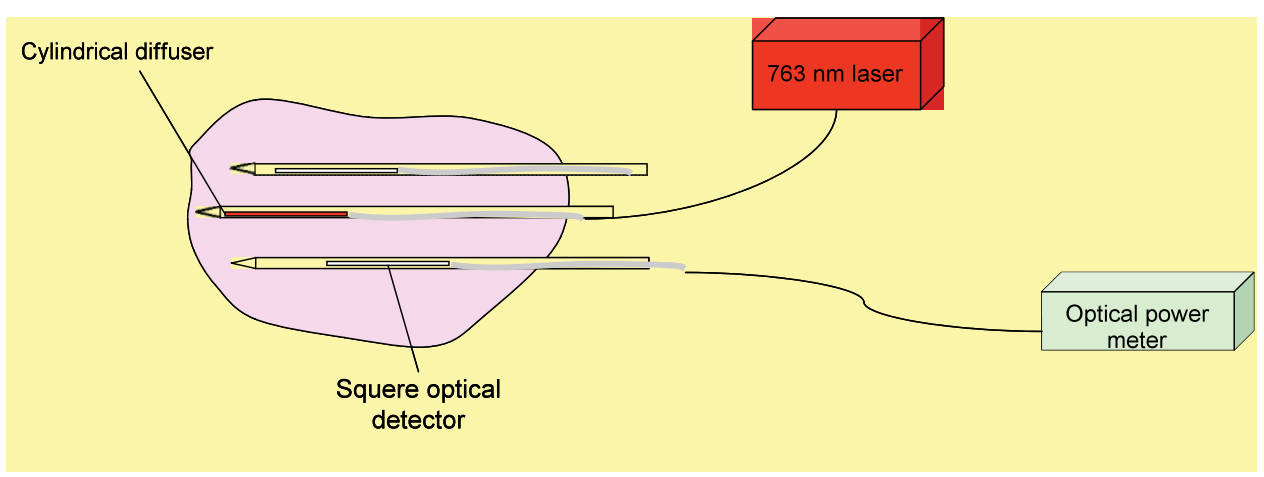

Fig. 2 Schematic diagram of equipment used for light delivery and detection.

fibre, i.e., less than the resolution of the measuring system at $0.2 \mathrm{~W}$. When the aiming beam is viewed with the fiber inside the needle, there are no visible variations in the translucency of the needle. This was confirmed by using a power meter (Newport Corporation, Irvine, CA), to measure the output of the fiber through the needle in one direction and then rotating the needle while keeping the fiber and the meter stationary.

The diffuser was placed within a needle and pushed in until it was approximately mid prostate, according to the prostate length measured on the CT scan. The detector was placed at the end of an adjacent or nearby needle, and then moved back through the prostate, with readings being taken at regular intervals. This was repeated with the detector in a different needle, until readings had been taken along all nearby needles in which light could be detected. The procedure was then repeated with the delivery fibre in another needle.

A dark reading was taken prior to switching on the laser. Once the laser was fired an optical reading was taken with the detector at the end of the needle. Readings were taken at $6 \mathrm{~mm}$ intervals (which corresponds to two CT slices), until the readings reached the dark reading. If any readings seemed unusually high or low, the series of readings along that detector needle were immediately repeated. The detector was taped to electronic callipers, mounted on a stand, to facilitate accurate movement.

The number of readings taken for each patient varied, depending on ease of accessibility of the needles and the length of time available for the study.

Once all the optical readings had been collected, the distances between the light delivery and light detector needles were

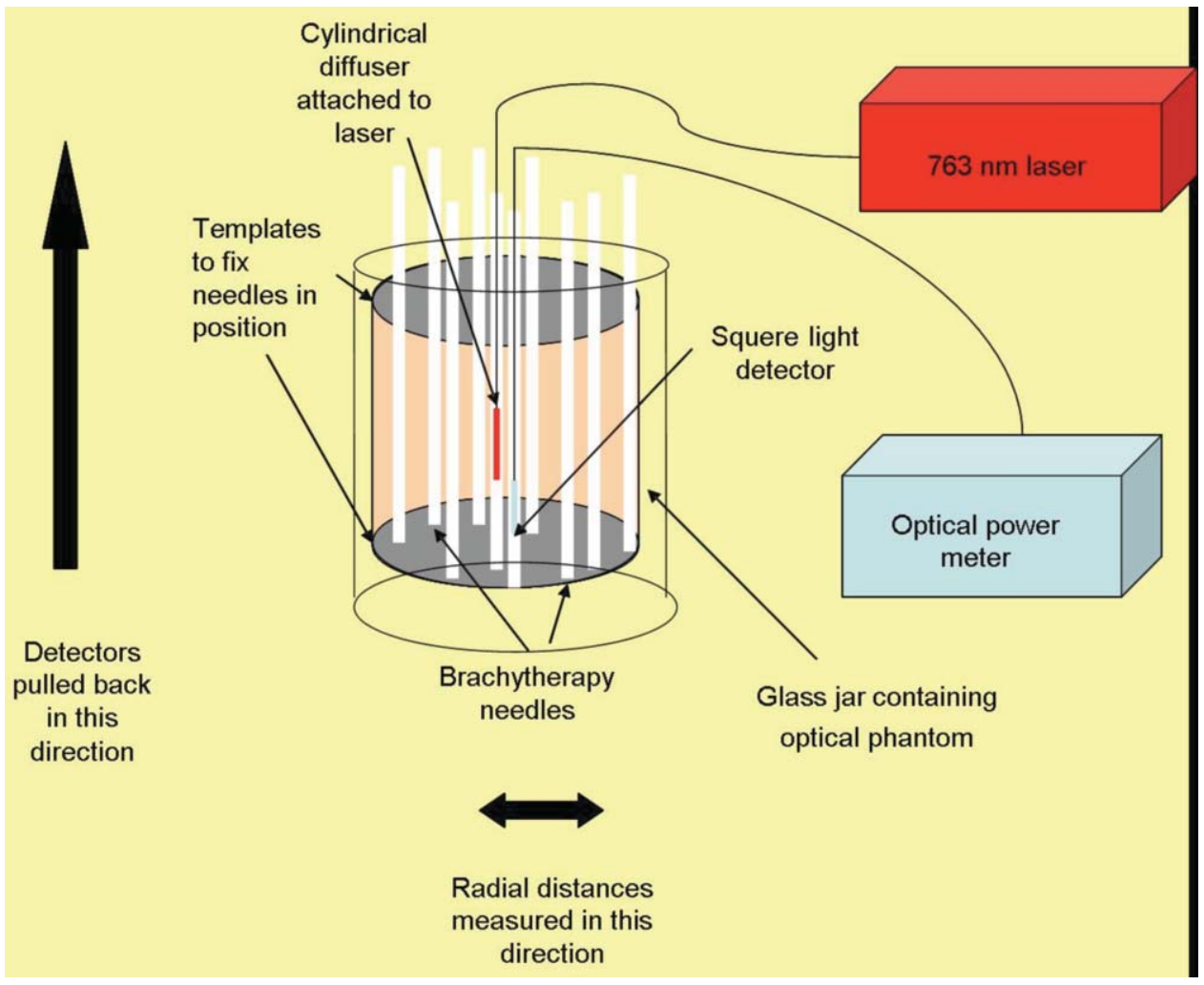

Fig. 3 Diagram of experimental set up for optical phantom. 
measured on the CT scan. As the needles are not exactly parallel, the distances between them were measured for each detector position, i.e., at $6 \mathrm{~mm}$ intervals along each track. Fluence readings from needles less than $3 \mathrm{~mm}$ apart were not used due to the fact that the diffusion approximation does not hold at short distances, and the errors in measuring the needle separation become highly significant.

\section{Calculation of Penetration Depth}

Pantelides described the use of the diffusion approximation to the Boltzmann equation to assess the effective attenuation coefficient of a medium based on multiple fluence readings taken at different distances from the source. ${ }^{14}$ The effective attenuation coefficient $\left(\mu_{\text {eff }}\right)$ is the inverse of the penetration depth (pd), which is the depth at which $67 \%$ of the light has been absorbed. For a cylindrical diffuser, the diffusion approximation to the Boltzmann equation can be expressed as:

$$
I_{(r)}=c_{1} I_{0}(r)^{1 / 2} e^{-r} \cdot \mu_{e f f},
$$

where $I_{r}$ is the fluence at radial distance $r$ from the diffuser, $I_{0}$ is the fluence at source, $\mathrm{r}$ is the radial distance in $\mathrm{cm}$ from the diffuser fiber, $\mu_{\text {eff }}$ is the effective attenuation coefficient for the volume of tissue between the source and $\mathrm{r}$, and $\mathrm{c}_{1}$ is a constant. By multiplying each side by $\mathrm{r} 1 / 2$ :

$$
I_{(r)}(r)^{1 / 2}=c_{1} I_{0} e-r \cdot \mu_{e f f} .
$$

Since $\mathrm{I}_{0}$ is constant, $\ln \left(\mathrm{c}_{1} \mathrm{I}_{0}\right)$ can be replaced by another constant $\mathrm{c}_{2}$ :

$$
\ln \left(I_{(r)}(r)^{1 / 2}=c_{2}-\mu_{e f f} \cdot r .\right.
$$

It can be seen that $\mu_{\text {eff }}$ is the negative slope of $\ln \left(\mathrm{I}_{\mathrm{r}} \mathrm{r}^{1 / 2}\right)$ plotted against radial distance. An advantage of this model is that it is not necessary to know the fluence at source. The model assumes that diffusion theory applies (i.e., the distance from the source is great enough for diffusion to have occurred, found experimentally to be $2-3 \mathrm{~mm}$ ). Diffusion theory assumes that the medium is homogenous and that the light source is an infinitely long cylinder. In this study the cylinder is only $2 \mathrm{~cm}$ long with the result that the fluence field around it is roughly ellipsoidal rather than cylindrical.The radial distance $r$ between the light delivery and light detector needle was measured on CT for each optical measurement position. In order to calculate the effective attenuation coefficient, a graph was plotted of the natural logarithm of the product of the fluence and the square root of the radial separation of the needles. For this, only the fluence reading at the mid point of the diffuser was used, for each of the detector needles.

\subsection{Validation of the optical technique}

This technique was validated using an intralipid and dye phantom, which was designed to have similar properties to previously published values for prostate tissue (based on Lees' data). [One liter of phantom was composed of $50 \mathrm{ml} 20 \%$ intralipid, $0.5 \mathrm{ml}$ dye (ICI 5109564, 5\% solution) with the balance being water, giving an effective attenuation coefficient $\left(\mu_{\mathrm{eff}}\right)$ of $0.173 \mathrm{~mm}^{-1}$ at $763 \mathrm{~nm}$. We gratefully acknowledge the help of Professor Hebden, Department of Medical Physics, UCL in undertaking this validation.]

The setup was validated using a container filled with the phantom, over which a custom-made template with 5-mm spaced holes was fitted (Fig. 3). The delivery needle was inserted into the phantom via a template hole, and the detector needles were positioned at various distances from the delivery needle. The cylindrical diffuser was positioned in a mid position in the phantom, and the detector was then withdrawn along the detector needle in $6 \mathrm{~mm}$ steps, which would correspond with a reading at every second CT slice in the clinical part of the study. Readings were taken of light intensity at each position.

\section{Results}

\subsection{Validation of the technique using an optical phantom}

Figure 4 shows the variation in fluence as the detector is moved in relation to the light delivery needle. Each line corresponds to the fluence readings along one nearby needle; the radial distance

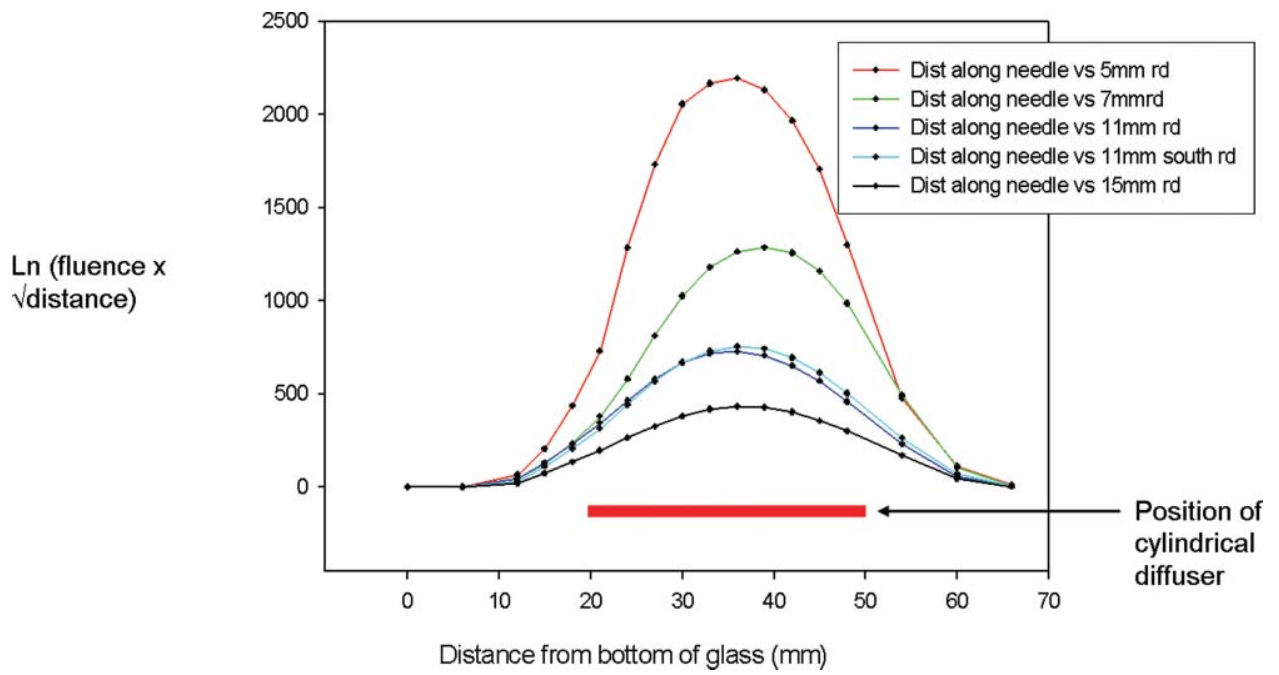

Fig. 4 Graph showing variation of light intensity with distance for a 2-cm cylindrical diffuser within a lipid phantom. 


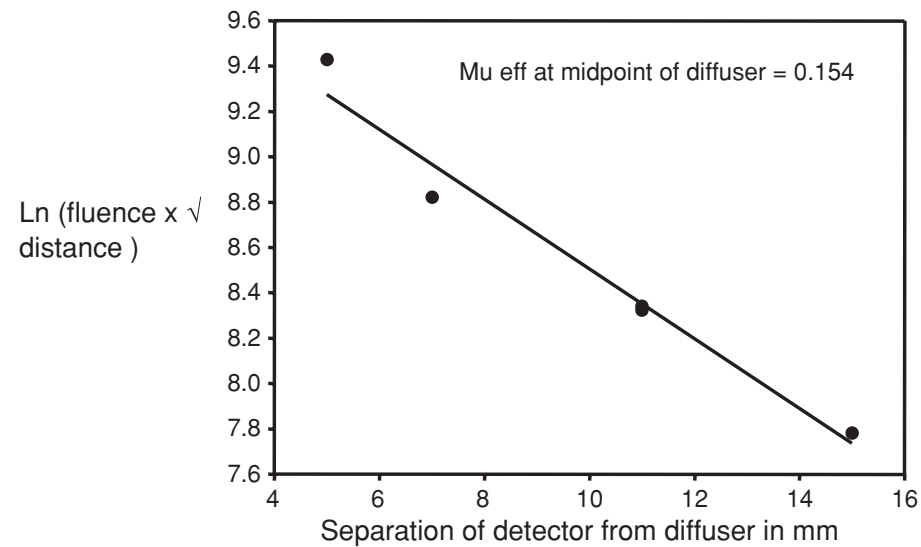

Fig. 5 Plot of $\ln$ (fluence $x \sqrt{ }$ distance) versus radial distance from the midpoint of the diffuser (at midpoint of diffuser on Fig. 4).

of the detector needle from the diffuser needle is shown in the legend. The fluence reduces with an increasing distance from the source, and the maximum fluence is recorded at the midpoint of the diffuser, for all needles except one (that at a $7 \mathrm{~mm}$ radial distance from the diffuser).

The graph shown in Fig. 5 is plotted from fluence measurements taken at the midpoint of the diffuser at four different radial distances, corresponding to the maximum fluence in four different needles. The position of the measurements is $33 \mathrm{~mm}$ from the bottom of the glass container, where the midpoint of the diffuser is expected to be.

The slope of this graph gives a value for $\mu_{\text {eff }}$ of 0.154 . The expected value from the phantom recipe was 0.173 . An experi- mental value within $11 \%$ of the predicted value is an acceptable experimental error for this kind of phantom. In the next section it can be seen that this discrepancy is small compared to the patient to patient or even the intra-prostatic variation in optical properties in individual patients.

\section{Clinical Results}

\subsection{Fluence readings along the detector needles}

The figures in this section show the variation in fluence along each detector needle for patient $\mathrm{F}$, who had the most readings taken. Figure 6 shows the variation in fluence along four different

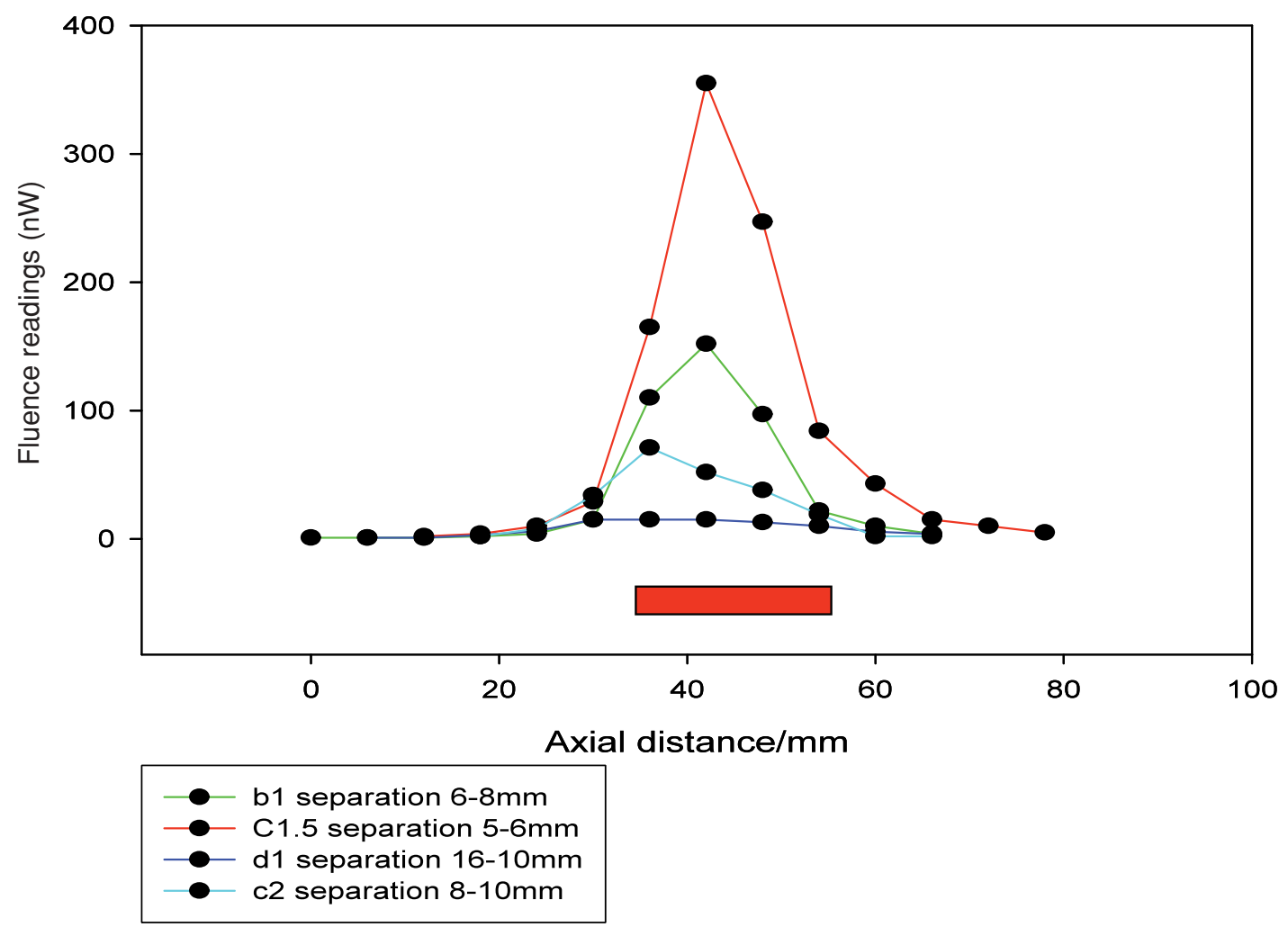

Fig. 6 Fluence readings along 4 different detectors as the detector is pulled back along needle (Patient F, source c1). 


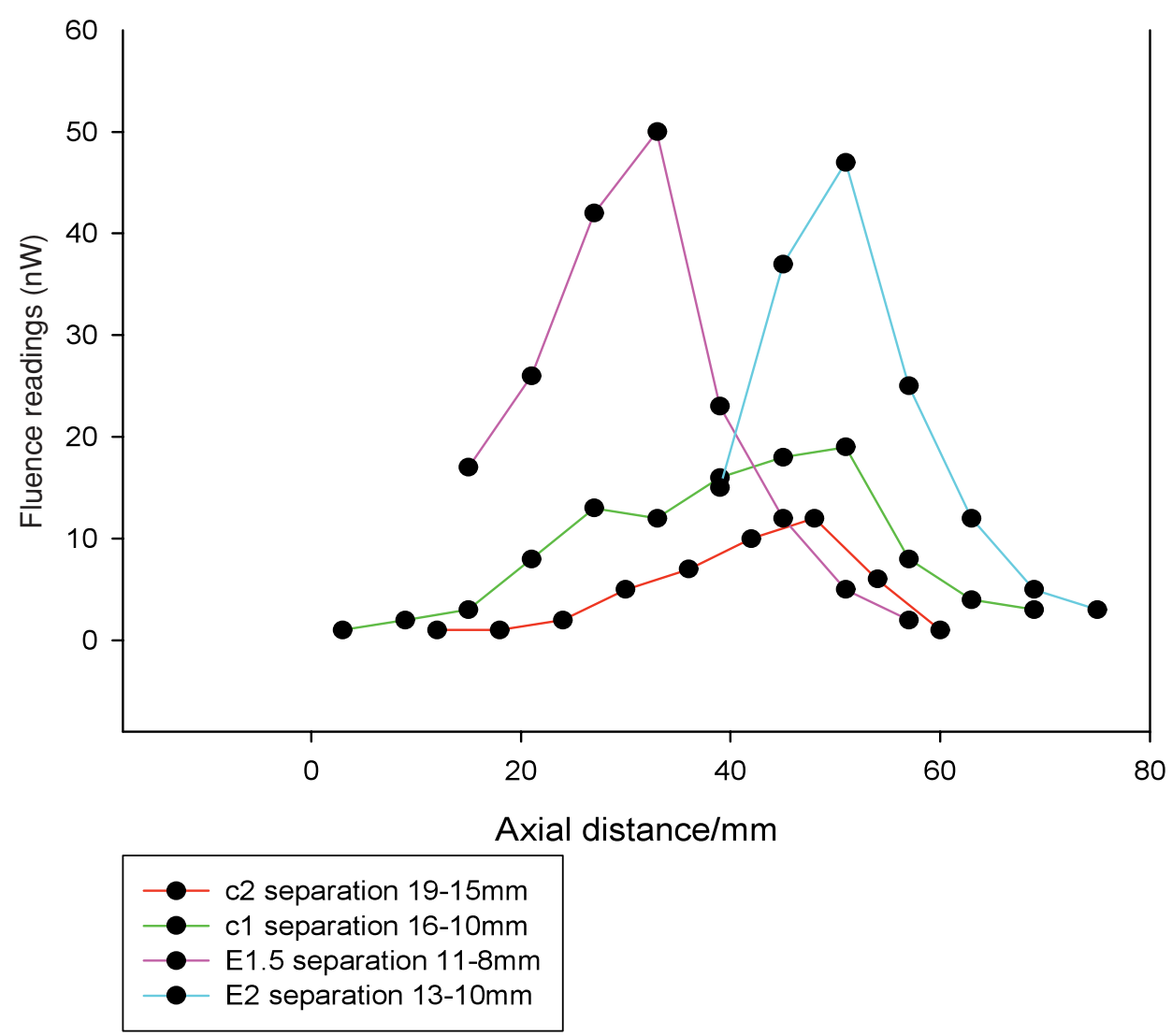

Fig. 7 Fluence readings as detector is pulled back along needle (Patient F, source d1).

needles at different distances from source at template position c1 (shown as a red bar underneath the plots). This graph looks similar to those obtained in the phantom study, with peak fluence detected by all detectors at the mid point of the source and greater fluence recorded in needles closer to the delivery fiber. However, not all of the sources showed fluence curves which were as predictable as those seen for the phantom.

Jumps in readings were quite common, and, as mentioned earlier, we immediately repeated these measurements whenever this was observed. Nevertheless, it can be seen that jumps were still visible, suggesting that it is not an experimental artifact.

For source d1 (Fig. 7) it can be seen that the maximum fluence is seen at different points along the diffuser, for two of the detector needles. This could be due to movement of the light delivery fibre between measurements made along one needle and measurements made along the next; also, however, it could be due to movement of the needle in which the light detector was placed, between the CT scan and the optical study. Another explanation would be that there was something (e.g., calcification) which deflected the light in one direction, giving a true higher reading at one position compared to another.

In addition, the needles were not always exactly parallel. This is reflected in the range of distances given in the legend for some light detector needles, such as source B1.5 (Fig. 8).

As the readings were recorded manually we were able to check them at the time, and those that are seen here are those that, if they did not follow an expected pattern, were rechecked and found to be the same on repeat testing. Therefore, the variation in light intensity seen here is a true reflection of that in the prostate at the time, within the limitations imposed by the needle positions, which were chosen for brachytherapy rather than for the optical study. This means that sometimes a fiber further away from the light delivery fiber will have higher fluence readings than one close to the needle.

\subsubsection{Effective attenuation coefficient and penetration depth}

The set of the plots used to calculate the $\mu_{\text {eff }}$, and hence the penetration depth (pd) for patient $\mathrm{F}$ is shown in Fig. 9. Each graph corresponds to one source position. The data points on the graph represent readings taken from needles at different radial distances from the source, using the readings taken closest to the midpoint of the diffuser fiber.

The gradient of each graph is the effective attenuation coefficient. The letters refer to the template position of the source. The penetration depth is the inverse of the effective attenuation coefficient.

Figure 10 shows the penetration depth schematically, for patients A, B, E, and F. The drawing shows the brachytherapy template, with letter coordinates on the $\mathrm{x}$ axis and number coordinates on the $y$ axis. Each template position can be identified by its letter and number coordinate, e.g. F1.5. An idealized prostate and urethra outline is drawn onto the template, for reference.

Each circle corresponds to one source. The centre of the circle is positioned at the template position for the source. The radius of the circle corresponds to the penetration depth, 


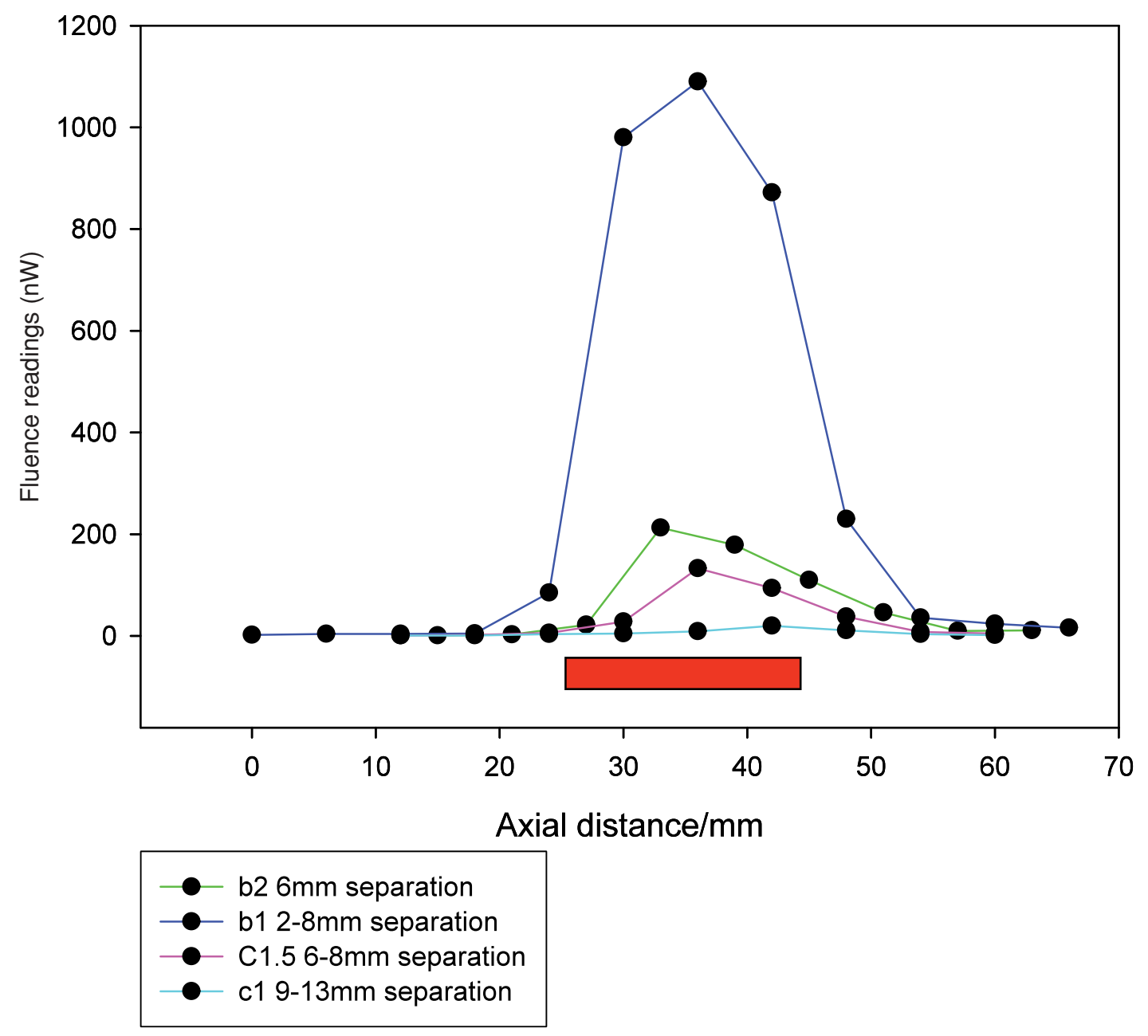

Fig. 8 Fluence readings as detector pulled back along needle (Patient F, source B1.5).

which is the inverse of the gradient of one of the graphs for that source.

Table 1 shows the $\mu_{\text {eff }}$ and pd (mean and range) for all patients. The variability factor was calculated for each patient as the ratio of the maximum to minimum pd. As can be seen from the table this variability factor ranged from 1.3 to 12.3 with a mean variability within an individual patient of 4.3 . It seems likely that this value of 12.3 fold variation in penetration depth in 1 patient (patient $\mathrm{E}$ ) was due to readings along one needle, and it is possible that these outlying values were artifactual error.

\section{Discussion}

\subsection{Methodological Limitations}

It can be seen from Fig. 4 which shows data from the phantom work that one of the detectors in the series (that at a $7 \mathrm{~mm}$ radial distance from the diffuser) showed an apparent peak of light intensity away from the midpoint of the diffuser. Although this may have been due to a difference in detected intensity at that point it is also possible that this was due to movement of the diffuser or detector from their intended positions. As this happened in the phantom it is also possible that this could have happened in the patient work, giving rise to error in our calculations.

Accurate measurement of the distances between the needles is required to give accurate estimates of the optical properties of the prostate. Zhu and colleagues presume a distance between needles based on perfect alignment of the needles with the template. ${ }^{5}$ Thus, measurement of the actual distances using CT slices at $6 \mathrm{~mm}$ intervals, as done in our study, should represent an improvement on the technique used by Zhu. However, the situation is complicated as the prostate swells following needle insertion, and the light fluence measurements were made 1-2 $\mathrm{h}$ after the CT scan, which was itself $1-2 \mathrm{~h}$ after the needles were inserted.

In order to overcome this problem, the light measurements could be made in the CT scanning room (which would be impractical as another patient would require the CT scanner), or measurements could be taken whilst the patient was under general anaesthetic, with needle separation measurements made on ultrasound. However, the idea of doing the study under anaesthetic was rejected on ethical grounds, as it would increase the total anaesthetic time, without benefit to the patient.

All the patients studied had had treatment with hormone therapy prior to brachytherapy. The effect of hormone treatment on 

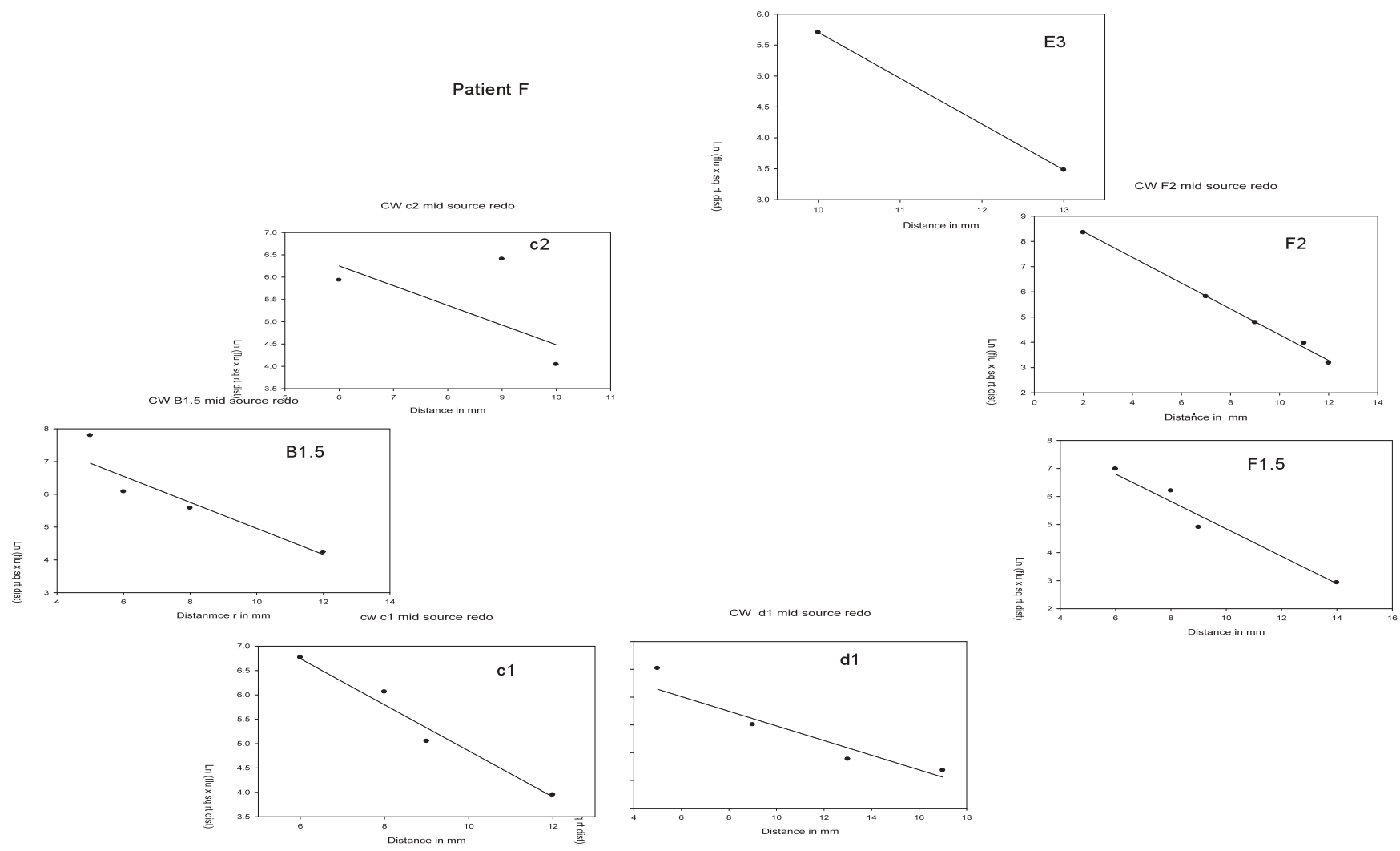

Fig. 9 Effective attenuation coefficient graphs for patient F.

the optical properties of the prostate in a human prostate cancer patient group is not known. However, as the prostate reduces in size, and, on needle insertion often feels tough in comparison to nonhormone treated prostate, it is certainly possible that the optical properties may change. Although the effect of hormone treatment limits the relevance of this study to the patients we have treated with PDT, it is possible that neoadjuvant hormone therapy may be considered in the PDT setting as well as the radiotherapy setting, and so we deemed it worthwhile to study this patient group. Because all men had had hormone therapy for at least 4 months prior to brachytherapy, it was not possible to assess for a difference between treatments of different duration.

The aim of our study was not to develop an optimal model of light fluence in tissues but to see whether dosimetry planning using optical measurements made intra-operatively could be used as the basis for dose planning. We therefore used a simple method for calculation of penetration depth, the same technique has been used by a number of other investigators however, Zhu used a more complex method of calculating penetration depth and noted similar variation to that seen in this study. ${ }^{5}$

Zhu also describes the use of a motorised probe to withdraw the detector within the needle, and software to record the optical readings, allowing many more readings to be taken than with our manual method. The similarity of our results would suggest that there may be little difference between the two methods.

Human prostate is composed of a number of different tissue types, including malignant and benign tissue, calcification, cysts and adenomas. The heterogeneity of prostate tissue is well demonstrated on dynamic contrast enhanced MRI. As this study used CT rather than MRI, it was not possible to determine whether the variation could be correlated with the different tissue types. Also, as we measured the penetration depth over relatively large prostate volumes, which overlapped, it would have been difficult to correlate the results with a particular tissue type. If both delivery and detector fibre were contained within the same open ended needle (in a technique similar to that used by Pantelides, Whitehurst, and Lee) and these needle positions were captured on MRI, then it may have been possible to correlate the tissue type and penetration depth. We could not have done this, however, as the patients already had closed ended needles in situ.

It is thought that bleeding around the needles could interfere with light distribution in the prostate. It is likely that his is a greater problem when using a shorter wavelength, such as the $630 \mathrm{~nm}$ used in other studies, where the specific extinction coefficient is $4.2 \mathrm{~cm}^{-1} \mathrm{mmole}^{-1}$. At the longer wavelength of $763 \mathrm{~nm}$ used in this work, haemoglobin has a specific extinction coefficient of $1.6 \mathrm{~cm}^{-1} \mathrm{mmole}^{-1}$ (Ref. 15) so the possible attenuating effect of blood pooling around the plastic needles should be less than that seen at $630 \mathrm{~nm}$. As we used CT images to assess the prostate difference it was not possible to detect bleeding, and hence to assess whether this directly affected the variability that we recorded. However, if the study was carried out using MRI, where blood is seen clearly on T1 weighted images, it would be possible to directly measure this.

In this study we have not determined conclusively whether the variability that we have shown is due to the prostate tissue itself, or bleeding around the needles, or indeed the equipment used. However, as can be seen from the discussion of the equipment the known variability of emission is around $\pm 3 \%$ over the 


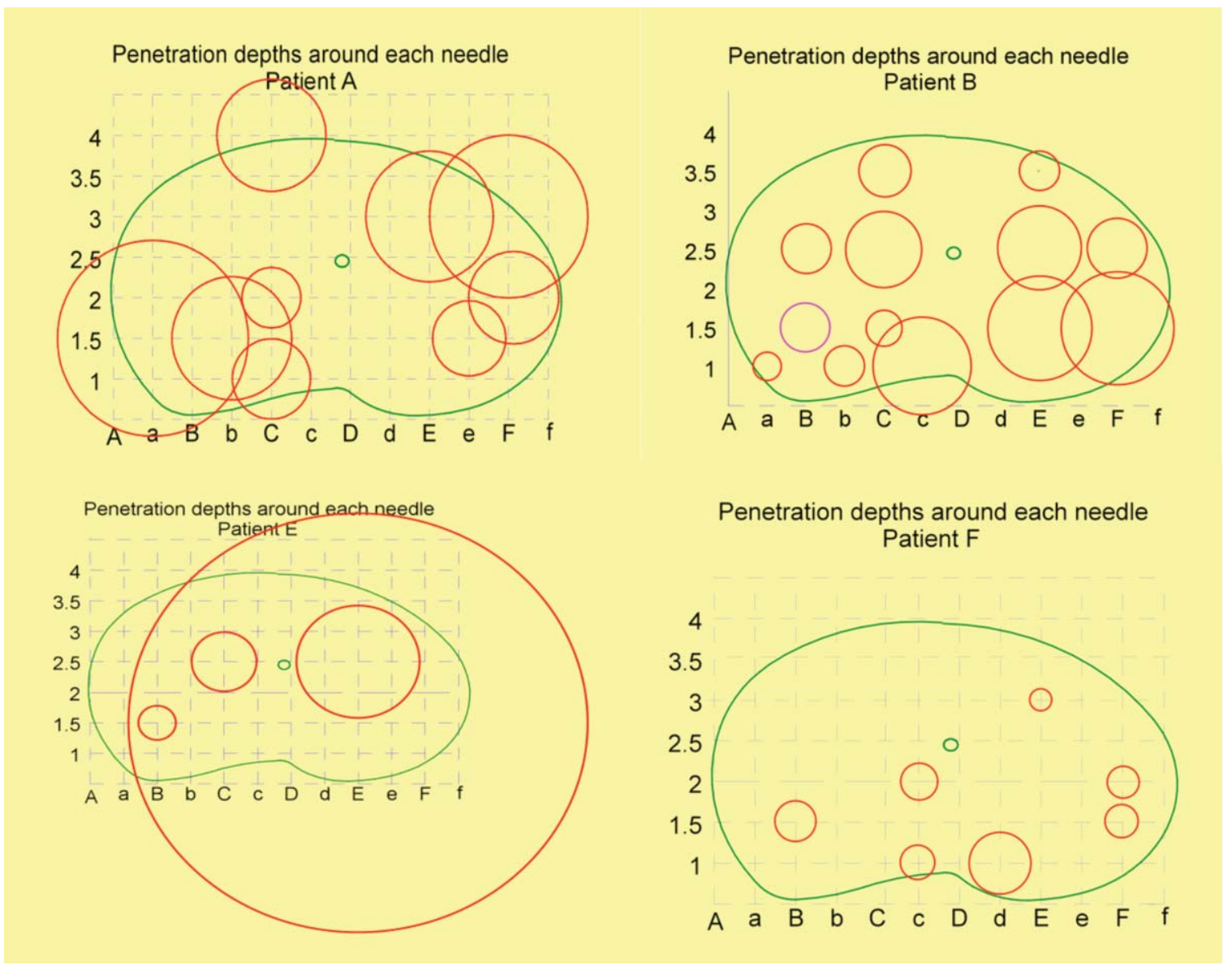

Fig. 10 i: Patient A penetration depth range $0.27-1.21 \mathrm{~cm}$ (variability factor 4.45); ii: patient B penetration depth $0.18-0.72 \mathrm{~cm}$; variability factor 3.96; iii: patient $\mathrm{E}$ penetration depth $0.28-3.43 \mathrm{~cm}$; variability factor 12.3; iv: patient $\mathrm{F}$ enetration depth $0.14-0.38 \mathrm{~cm}$; variability factor 2.81 .

central position of the diffuser where the optical properties were calculated from, and that the detector variability is within the order of $\pm 10 \%$. The variability in calculated optical properties that we recorded was much greater than this, and so it is likely that this is either due to prostate variability or bleeding around the needles. However, the object of this study was to see if it would be useful to calculate optical properties using data which could be collected during clinical prostate PDT; in this situation it would not be possible to eliminate, or assess, bleeding around the needles. So, whatever the cause of the variability we

Table 1 Effective attenuation coefficient ( $\mu_{\text {eff }}$ ) and penetration depth (pd) for each patient.

\begin{tabular}{lcccccccccc}
\hline Patient & $\begin{array}{c}\text { No. of } \\
\text { readings }\end{array}$ & $\begin{array}{c}\text { No. of } \\
\text { needle pairs }\end{array}$ & $\begin{array}{c}\text { No of } \\
\text { sources }\end{array}$ & $\begin{array}{c}\text { Mean } \mu_{\text {eff }} \\
\left(\mathrm{cm}^{-1}\right)\end{array}$ & $\begin{array}{c}\text { Minimum } \mu_{\text {eff }} \\
\left(\mathrm{cm}^{-1}\right)\end{array}$ & $\begin{array}{c}\text { Maximum } \mu_{\text {eff }} \\
\left(\mathrm{cm}^{-1}\right)\end{array}$ & $\begin{array}{c}\text { Minimum } \\
\mathrm{pd}(\mathrm{cm})\end{array}$ & $\begin{array}{c}\text { Maximum } \\
\mathrm{pd}(\mathrm{cm})\end{array}$ & $\begin{array}{c}\text { Mean } \\
\mathrm{pd}(\mathrm{cm})\end{array}$ & $\begin{array}{c}\text { Variability factor } \\
(\mathrm{max} / \mathrm{min} \mathrm{pd})\end{array}$ \\
\hline A & 31 & 31 & 10 & 1.51 & 0.83 & 3.69 & 0.27 & 1.21 & 0.66 & 4.45 \\
B & 312 & 54 & 13 & 2.34 & 1.38 & 5.46 & 0.18 & 0.724 & 0.43 & 3.96 \\
C & 90 & 10 & 2 & 1.75 & 1.53 & 2.03 & 0.49 & 0.655 & 0.57 & 1.33 \\
D & 116 & 21 & 5 & 1.81 & 1.20 & 3.38 & 0.30 & 0.835 & 0.55 & 2.82 \\
E & 87 & 11 & 4 & 0.78 & 0.29 & 3.57 & 0.28 & 0.343 & 1.28 & 12.3 \\
F & 286 & 29 & 7 & 4.36 & 2.63 & 7.41 & 0.14 & 0.379 & 0.23 & 2.81 \\
G & 68 & 9 & 3 & 2.96 & 1.68 & 5.43 & 0.18 & 0.596 & 0.34 & 3.24 \\
H & 87 & 14 & 4 & 2.06 & 0.95 & 4.65 & 0.22 & 1.05 & 0.49 & 4.87 \\
Mean & 134 & 22.3 & 6 & 1.76 & 0.91 & 3.89 & 0.26 & 1.11 & 0.57 & 4.32 \\
\hline
\end{tabular}




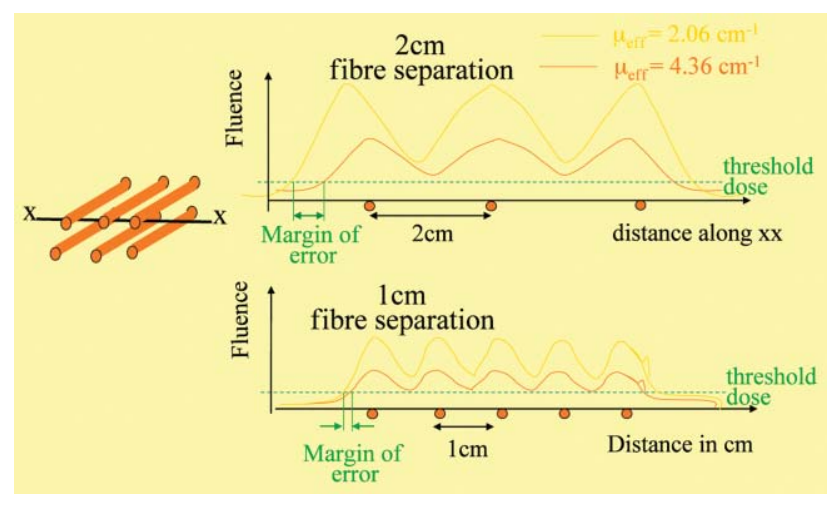

Fig. 11 Effect of fiber separation on absolute fluence levels.

postulate that this would also occur in the clinical prostate PDT setting. It may be that the way to overcome this is to give light doses well above the threshold required, so that the variability can be compensated for.

\subsection{Comparison of Data to Other Studies}

The closest results to those of this study are those reported by Zhu using multiple measurements prior to light delivery for PDT using motexafin lutetium. ${ }^{5}$ Zhu used the wavelength $(732 \mathrm{~nm})$ closest to that which we used, and, like us, also took multiple measurements throughout the prostate. He reports a similar range of values for penetration depth, with similar variability to our data. The large variability, in comparison to work done by Lee and colleagues may well be due to intrinsic variability in the optical properties of prostate tissue, which is detected by the larger number of measurements taken. The mean values for a range of studies are shown in Table 2, with data from this study also included.

Svensson and colleagues from Lund report variation between values derived using the diffusion approximation, and the novel White Monte Carlo approach that they use $\mathrm{s}^{7,16,17}$ The fibre separation distances used in this work were much greater than those that we used, and would not be expected to be able to detect a variation in optical properties at smaller distances.

\subsubsection{Results from the Toronto group using the same wavelength as this study}

The Toronto group ${ }^{22}$ used the same wavelength as that used in our study, as they were investigating the use of the same photosensitizer. Their results show a mean $\mu_{\mathrm{eff}}$ of $2.0 \pm 0.6 \mathrm{~cm}^{-1}$, which is similar to the $\mu_{\text {eff }}$ in this study of $1.76 \mathrm{~cm}^{-1}$. The differences in the two studies lies in the tissue evaluated (treatment naïve in this study and post radiotherapy in the Toronto study) and in the method of calculation of the optical properties.

\subsubsection{Implications of results for photodynamic therapy}

If the tissues in a prostate lobe were homogenous, then it would in principle be possible to measure the optical properties of that lobe at the beginning of the PDT procedure and adjust the power of the light source and the treatment time accordingly. However it appears that the lobes are far from optically homogenous.
These results are given in terms of penetration depth, which is the distance at which $67 \%$ of the light intensity is lost. If the energy delivered to each needle was smaller, and a larger number of needles were used, then the absolute variability in fluence throughout the prostate would be reduced. It is the absolute fluence level in any given volume of tissue which is likely to determine necrosis (as well as the levels of drug and oxygen available at the same time).

This is shown schematically in Fig. 11, for needle separation of 10 and $20 \mathrm{~mm}$ for two different $\mu_{\mathrm{eff}}$ : $\left(\mu_{\mathrm{eff}}\right.$ : of $4.4 \mathrm{~cm}^{-1}$, mean value for patient $\mathrm{F}$ and $\mu_{\mathrm{eff}}:$ of $2.1 \mathrm{~cm}^{-1}$ mean value for patient $\mathrm{H})$.

Calculation of the number of needles required to achieve appropriate light distribution throughout the prostate, and the optimum positioning of those needles would require a complex plan, based on the optical properties in different volumes of the prostate.

Altschuler, working with Zhu and others has used the Cimmino feasibility algorithm to optimize prostate PDT treatment planning. ${ }^{18}$ For the purpose of their calculations they used a standard 12 light delivery fibers. This model was based on homogenous drug distribution, and homogenous optical properties,

Table 2 Comparison of $\mu_{\text {eff }}$ and pd in different studies.

\begin{tabular}{|c|c|c|c|}
\hline Reference & $\begin{array}{l}\text { Wavelength } \\
(\mathrm{nm})\end{array}$ & $\begin{array}{c}\text { Mean } \\
\text { penetration } \\
\text { depth }(\mathrm{cm})\end{array}$ & $\begin{array}{c}\text { Mean } \\
\text { effective } \\
\text { attenuation } \\
\text { coefficient } \\
\left(\mathrm{cm}^{-1}\right)\end{array}$ \\
\hline \multirow[t]{4}{*}{ Pantelides, $1990^{14}$} & 633 & 0.22 & 4.3 \\
\hline & 612 & 0.16 & 6.25 \\
\hline & 594 & 0.11 & 9.09 \\
\hline & 543 & 0.055 & 18.1 \\
\hline Chen, $1993^{24}$ & 630 & 0.225 & 4.44 \\
\hline \multirow[t]{2}{*}{ Whitehurst, $1994^{2}$} & 633 & $\begin{array}{c}\text { Prostate } \\
\text { cancer } \\
0.27\end{array}$ & $\begin{array}{c}\text { Prostate } \\
\text { cancer } 3.6\end{array}$ \\
\hline & & $\begin{array}{c}\text { Benign } \\
\text { tissue } 0.29\end{array}$ & $\begin{array}{l}\text { Benign } \\
\text { tissue } 3.5\end{array}$ \\
\hline \multirow[t]{2}{*}{ Lee, $1995^{3}$} & 633 & $0.31 \mathrm{~cm}$ & 3.2 \\
\hline & 665 & $0.26 \mathrm{~cm}$ & 3.9 \\
\hline Lee, $1999^{4}$ & 633 & 0.29 & 3.5 \\
\hline Zhu, $2005^{5}$ & 730 & 0.4 & 3.3 \\
\hline Weersink, $2005^{23}$ & 762 & 0.5 & 2.0 \\
\hline \multirow[t]{3}{*}{ Svensson, $2007^{17}$} & 660 & 0.28 & 3.6 \\
\hline & 786 & 0.35 & 2.9 \\
\hline & 916 & 0.26 & 3.8 \\
\hline These data & 763 & 0.56 & 1.76 \\
\hline
\end{tabular}


at either the mean or maximum of their measured variables. It is thus based on a geometric model. They achieved more conformal dose plans with this algorithm than with the standard dose plan. However, whether this translates into more conformal prostate necrosis is not reported, and this group have not reported imaging findings, e.g., MRI on which treatment effect could be assessed and compared to the fluence distribution.

More recent work by $\mathrm{Li}$ and $\mathrm{Zhu}^{11}$ has shown that use of heterogeneous optical properties for comprehensive optimisation of source parameters (strength, length, and location) is feasible in a clinical timeframe (less than $3 \mathrm{~min}$ ), and allows acceptable dose coverage of a heterogeneous prostate. This work used 3D distributions of optical properties in a patient prostate to obtain a more accurate light fluence kernel, which, for specified sources and points gives the fluence delivered to a point by a source of unit strength. This kernel was then used to solve the inverse problem of determination of the sources and strengths needed to deliver a given light fluence distribution during PDT. Two different kernels were used in order to explore the use of a simpler algorithm with a faster solution and a more accurate but slower algorithm. The Cimmino feasibility algorithm is used to restrict the optimisation process to given sources and determine the appropriate source strengths. They compared the plan reported previously using homogenous optical properties and the Cimmino algorithm ${ }^{18}$ to that derived from heterogeneous optical properties and found that use of heterogeneous properties resulted in a treatment plan with a more homogenous fluence distribution, achieved by changing the light source positions as well as strengths.

Davidson and colleagues have reported their work in treatment planning and dose analysis using the photosensitizer Tookad in men with recurrent prostate cancer following radiotherapy. ${ }^{19}$ The clinical aspects of the study are reported separately. ${ }^{20-23}$ They used treatment planning software based on predicted light distributions to plan PDT treatment, and then used the same software to reconstruct the treatment delivered, to evaluate the accuracy of the predictions, and to evaluate the concept of threshold light dose, in a similar approach to that used in radiotherapy. They were able to assign a threshold light dose of 23 joules per square centimeter; if $90 \%$ of the prostate received a light dose above that, then $62 \%$ of patients were biopsy negative at 6 months; none of the men with $90 \%$ of the prostate treated below that level had a negative biopsy at 6 months. For the treatment planning, however, they assumed homogenous optical properties. It is difficult to know whether the greater homogeneity in the optical properties that they found was due to a difference in the prostate after radiotherapy compared to the pre radiotherapy prostate that we studied, or whether it could be explained by the difference in techniques of optical measurement and analysis.

\section{Conclusions}

This study has shown a mean of four fold variability in light penetration at $763 \mathrm{~nm}$ in the human prostate, with one patient showing 12 -fold variability. The high degree of variability suggests that, for clinical studies of prostate PDT a large number of needles should be used with overlapping zones of illumination. In addition, planning based on a priori estimates of optical properties is likely to be very inaccurate, and difficult to carry out in the clinical setting. Even planning based on real time measurements of the tissues will be difficult because the optical properties vary rapidly over small distances so that a large number of measurements would have to be made to map the region to be treated.

It is only possible to determine whether this variability in light penetration would result in a similar variability in photodynamic therapy effect, in a clinical study of prostate photodynamic therapy, where photodynamic therapy effect is correlated with optical properties throughout the gland.

\section{References}

1. A. Jemal, R. Siegel, E. Ward, Y. Hao, J. Xu, and M. J. Thun, "Cancer statistics," CA Cancer J. Clin. 59(4), 225-249 (2009).

2. C. Whitehurst, M. L. Pantelides, J. V. Moore, P. J. Brooman, and N. J. Blacklock, "In vivo laser light distribution in human prostatic carcinoma," J. Urol. 151(5),1411-1415 (1994).

3. L. K. Lee, C. Whitehurst, M. L. Pantelides, and J. V. Moore, "In situ comparison of $665 \mathrm{~nm}$ and $633 \mathrm{~nm}$ wavelength light penetration in the human prostate gland." Photochem. Photobiol. 62(5), 882-886 (1995).

4. L. K. Lee, C. Whitehurst, M. L. Pantelides, and J. V. Moore, "An interstitial light assembly for photodynamic therapy in prostatic carcinoma," BJU Int. 84(7), 821-826 (1999).

5. T. C. Zhu, A. Dimofte, J. C. Finlay, D. Stripp, T. Busch, J. Miles, et al., "Optical properties of human prostate at $732 \mathrm{~nm}$ measured in mediated photodynamic therapy," Photochem. Photobiol. 81(1), 96-105 (2005).

6. K. K. Wang, T. Fau, and T. C. Zhu, "Reconstruction of in-vivo optical properties for human prostate using interstitial diffuse optical tomography," Opt. Express 17(14), 11665-11672 (2009).

7. T. F. Svensson, E. Alerstam, M. Einarsdottir, M. F. Einarsdottir, K. F. Svanberg, S. Andersson-Engels S "Towards accurate in vivo spectroscopy of the human prostate," J. Biophotonics 1(3), 200-203 (2008).

8. A. Dimofte, J. C. Finlay, and T. C. Zhu, "A method for determination of the absorption and scattering properties interstitially in turbid media," Phys. Med. Biol. 50(10), 2291-2311 (2005).

9. J. F. Axelsson, J. F. Swartling, and S. Andersson-Engels, "In vivo photosensitizer tomography inside the human prostate," Opt. Lett. 34(3), 232-234 (2009).

10. C. M. Moore, D. Pense, and M. Emberton "Photodynamic therapy for prostate cancer-a review of current status and future promise," Nat. Clin. Pract. Urol. 6(1), 18-30 (2009).

11. J. Li, M. D. Altschuler, S. M. Hahn, and T. C. Zhu, "Optimization of light source parameters in the photodynamic therapy of heterogeneous prostate," Phys. Med. Biol. 53(15), 4107-4121 (2008).

12. G. Yu, T. Durduran, C. Zhou, T. C. Zhu, J. C. Finlay, T. M. Busch, S. B. Malkowicz, S. M. Hahn, and A. G. Yodh, "Real-time in situ monitoring of human prostate photodynamic therapy with diffuse light," Photochem. Photobiol. 82(5), 1279-1284 (2006).

13. T. C. Zhu, J. Finlay, and S. Hahn, "Determination of the distribution of light, optical properties, drug concentration, and tissue oxygenation in-vivo in human prostate during motexafin lutetium-mediated photodynamic therapy," J. Photochem. Photobiol. B 79(3), 231-241 (2005).

14. C. Whitehurst, M. L. Pantelides, J. V. Moore, and N. J. Blacklock, "Photodynamic therapy for localised prostatic cancer: light penetration in the human prostate gland," J. Urol. 143(2), 398-401 (1990).

15. W. G. Zijlstra, A. Buursma, and O. W. van Assendelft, Visible and Near Infrared Absorption Spectra of Human and Animal Haemoglobin Chapter 7: Determination of absorption spectra, pp. 47-51, VSP, Utrecht (2000).

16. E. Alerstam, S. Andersson-Engels, and T. Svensson, "White Monte Carlo for time-resolved photon migration," J. Biomed. Opt. 13(4), 041304 (2008).

17. T. Svensson, E. Alerstam, M. Einarsdóttír, K. Svanberg, and S. Andersson-Engels, "In vivo optical characterization of human prostate tissue using near-infrared time-resolved spectroscopy," J. Biomed. Opt. 12(1); 014022 (2007).

18. M. D. Altschuler, T. C. Zhu, J. Li, and S. M. Hahn, "Optimized interstitial PDT prostate treatment planning with the Cimmino feasibility algorithm," Med. Phys. 32(12); 3524-3536 (2005). 
19. S. R. Davidson, R. A. Weersink, M. A. Haider, M. R. Gertner, A. Bogaards, D. Giewercer, A. Scherz, M. D. Sherar, M. Elhilali, J. L. Chin, J. Trachtenberg, and B. C. Wilson, " Treatment planning and dose analysis for interstitial photodynamic therapy of prostate cancer," Phys. Med. Biol. 54(8), 2293-2313 (2009).

20. M. A. Haider, S. R. Davidson, A. V. Kale, R. A. Weersink, A. J. Evans, A. Toi, M. R. Gertner, A. Bogaards, B. C. Wilson, J. L. Chin, M. Elhilali, and J. Trachtenberg, "Prostate gland: MR imaging appearance after vascular targeted photodynamic therapy with palladium-bacteriopheophorbide," Radiology 244(1), 196-204 (2007).

21. J. Trachtenberg, R. A. Weersink, S. R. Davidson, M. A. Haider, A. Bogaards, M. R. Gertner, A. Evans, A. Scherz, J. Savard, J. L. Chin, B. C. Wilson, and M. Elhilali, "Vascular-targeted photodynamic therapy (padoporfin, WST09) for recurrent prostate cancer after failure of external beam radiotherapy: a study of escalating light doses," BJU Int 102(5), 556-562 (2008).
22. J. Trachtenberg, A. Bogaards, R. A. Weersink, M. A. Haider, A. Evans, S. A. McCluskey, A. Scherz, M. R. Gertner, C. Yue, S. Appu, A. Aprikian, J. Savard, B. C. Wilson, and M. Elhilali, "Vascular targeted photodynamic therapy with palladium-bacteriopheophorbide photosensitizer for recurrent prostate cancer following definitive radiation therapy: assessment of safety and treatment response," J. Urol. 178(5), 1974-1979 (2007).

23. R. A. Weersink, A. Bogaards, M. Gertner, S. R. Davidson, K. Zhang, G. Netchev, J. Trachtenberg, and B. C. Wilson, “ Techniques for delivery and monitoring of TOOKAD (WST09)-mediated photodynamic therapy of the prostate: clinical experience and practicalities," J. Photochem. Photobiol. B 79(3), 211-222 (2005).

24. Q. Chen, S. D. Shetty, L. Heads, F. Bolin, B. Wilson, M. Patterson, L. Sirls, D. Schultz, J. C. Cerny, F. W. Hetzel, Photodynamic therapy in prostate cancer: optical dosimetry and response of normal tissue. SPIE 1881 Optical methods for tumour treatment and detection, 231-235 1993 (Conference proceeding). 